\title{
Approximation treatment for Linear Fuzzy HIV Infection Model by Variational Iteration Method
}

\author{
Hafed H Saleh ${ }^{1}$, Azmi A. ${ }^{1}$, Ali. F. Jameel ${ }^{2, *}$ \\ ${ }^{1}$ School of Mathematical Sciences, Universiti Sains Malaysia, 11800 USM, Penang, Malaysia \\ ${ }^{2}$ School of Quantitative Sciences, College of Arts and Sciences, Universiti Utara Malaysia, 06010 UUM Sintok, Kedah, Malaysia
}

Received November 16, 2020; Revised March 11, 2021; Accepted March 21, 2021

\begin{abstract}
Cite This Paper in the following Citation Styles
(a): [1] Hafed H Saleh, Azmi A., Ali. F. Jameel, "Approximation treatment for Linear Fuzzy HIV Infection Model by Variational Iteration Method," Mathematics and Statistics, Vol. 9, No. 3, pp. 342 - 349, 2021. DOI: 10.13189/ms.2021.090316.
\end{abstract}

(b): Hafed H Saleh, Azmi A., Ali. F. Jameel (2021). Approximation treatment for Linear Fuzzy HIV Infection Model by Variational Iteration Method. Mathematics and Statistics, 9(3), 342 - 349. DOI: 10.13189/ms.2021.090316.

Copyright $\bigcirc 2021$ by authors, all rights reserved. Authors agree that this article remains permanently open access under the terms of the Creative Commons Attribution License 4.0 International License

\begin{abstract}
There has recently been considerable focus on finding reliable and more effective approximate methods for solving biological mathematical models in the form of differential equations. One of the well-known approximate or semi-analytical methods for solving linear, nonlinear differential well as partial differential equations within various fields of mathematics is the Variational Iteration Method (VIM). This paper looks at the use of fuzzy differential equations in human immunodeficiency virus (HIV) infection modeling. The main advantage of the method lies in its flexibility and ability to solve nonlinear equations easily. VIM is introduced to provide approximate solutions for linear ordinary differential equation system including the fuzzy HIV infection model. The model explains the amount of undefined immune cells, and the immune system viral load intensity intrinsic that will trigger fuzziness in patients infected by HIV. CD4+T-cells and cytototoxic T-lymphocytes (CTLs) are known for the immune cells concerned. The dynamics of the immune cell level and viral burden are analyzed and compared across three classes of patients with low, moderate and high immune systems. A modification and formulation of the VIM in the fuzzy domain based on the use of the properties of fuzzy set theory are presented. A model was established in this regard, accompanied by plots that demonstrate the reliability and simplicity of the methods. The numerical results of the model indicate that this approach is effective and easily used in fuzzy domain.
\end{abstract}

Keywords Fuzzy Set Theory, Fuzzy Differential Equations, Fuzzy HIV Infection Model, Analytical
Solution, Variational Iteration Method (VIM)

\section{Introduction}

The mathematical model can be interpreted in many real dynamics of a set of ordinary or partial differential equations. Real life systems use fuzzy sense to generalize the common sense, as the fuzzy logic is a solid method to model vagueness and, in particular, to handle uncertainty by a mathematical model [1]. Since fuzzy differential equations (FDEs) are better known for their appropriateness to solve scientific and engineering problems, they have been a popular theme studied by a number of researchers recently. There are many practical problems with the solutions of the FDEs that meet fading initial or boundary value conditions problems. From many HIV models classified as a system of differential equations of first order. A Number of modeling methods for understanding dynamics of HIV, disease progression, anti -retroviral response, etc. have been proposed in [2-4]. The topic of FDEs has become a popular subject among mathematical scientists as it can be used in many branches, such as physics, astronomy, biology and population [5-7]. Such equation models are cell formation, population dynamics and HIV dynamics [8]. Given the approximate solutions to fuzzy initial value problems (FIVPs), many of these are not solved precisely and sometimes the exact solutions might not be available under uncertainty 
properties [9]. Also the approximate methods have advantages over numerical methods such as the flexibility to provide both linear and nonlinear problems with approximate and reliable solutions without any need for discretization and linearization and helps engineers or scientists to understand a physical problem better, and improving future methods and principles for solving their problems. The VIM is an approximate method created by $\mathrm{He}$ and applied widely in many mathematics problems and engineering applications [10-12]. Moreover, in the last years, VIM have been used to find the approximate solution of a different order of FDEs involving linear and nonlinear problems [13-15]. In this work, by means of a linear FDE system, the unpredictable behaviours of CD4 + T-cells, CTLs [16] and the viral load in different patients are modelled. Following this, the optimal control is analyzed for reducing both the viral load and the drug costs to a minimum value. Therefore, for this reason, the VIM will be applied to the solution of the proposed linear fuzzy HIV infection model. The approach is developed using the fuzzy set theory properties in the study of the estimated solution for CD4 + T-cells and CTL level and the viral load in various patients.

\section{Preliminaries}

Definition 2.1 [17]. Fuzzy numbers represent unknown values and are a subset of the set of real numbers. Fuzzy numbers are connected to membership degrees that state how valid it is to tell whether or not anything belongs to a given set. If identified by three numbers $\alpha<\beta<\gamma$, a fuzzy number $\mu$ is termed a triangular fuzzy number where $\mu(x)$ is a triangle centered on the $[\alpha, \gamma]$ interval and vertex at the point $x=\beta$. The following form is used for its membership function:

$$
\mu(x ; \alpha, \beta, \gamma)=\left\{\begin{aligned}
0, & \text { if } x<\alpha \\
\frac{x-\alpha}{\beta-\alpha}, & \text { if } \alpha \leq x \leq \beta \\
\frac{\gamma-x}{\gamma-\beta}, & \text { if } \beta \leq x \leq \gamma \\
0, & \text { if } x>\gamma
\end{aligned}\right.
$$

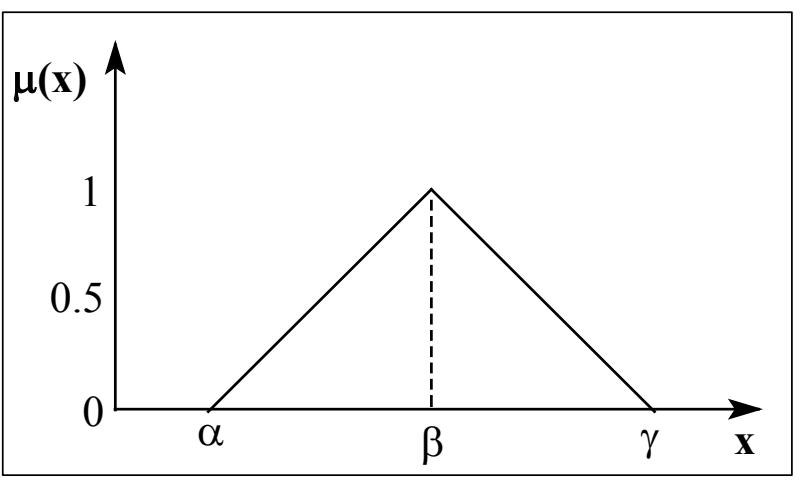

Figure 1. Triangular Fuzzy Number and its $r$-level is: $[\mu]_{r}=[\alpha+r(\beta-\alpha), \gamma-r(\gamma-$ $\beta)], r \in[0,1]$.The $r$-level set of trapezoidal fuzzy number can be defined as follows: $[\tilde{\mu}]_{r}=[(\beta-\alpha) r+\alpha, \delta-$ $(\delta-\gamma) r]$.

In this paper, $\tilde{E}$ indicates the class of all fuzzy subsets of $\mathbb{R}$ and fulfill the following properties [18]:

1. $\mu(x)$ is normal, i.e. $\exists t_{0} \in \mathbb{R}$ with $\mu\left(t_{0}\right)=1$,

2. $\mu(x)$ is fuzzy convex set, i.e. $\mu(\lambda x+(1-\lambda) t) \geq$ $\min \{\mu(x), \mu(t)\} \forall x, t \in \mathbb{R}, \lambda[0,1]$,

3. $\mu$ is upper semi-continuous on $\mathbb{R}$,

4. $\overline{\{t \in \mathbb{R}: \mu(x)>0\}}$ is compact.

The space of fuzzy numbers is called $\tilde{E}$ and $\mathbb{R}$ is a proper subset of $\tilde{E}$.

The $r$-level set is defined as $x \in \mathbb{R},[\mu]_{r}=\{x$ : $\mu(x) \geq r\}, 0 \leq r \leq 1$, where $[\mu]_{0}=\{x: \mu(x)>0\}$ is compact, and an interval that is closed bounded and denoted by $[\mu] \operatorname{rr}[\mu]_{r}=[\underline{\mu}(x), \bar{\mu}(x)]$. A fuzzy number is defined by an ordered pair of functions $[\underline{\mu}(x), \bar{\mu}(x)]$, $r \in[0,1]$ in a single parametric form which satisfies:

1. $\mu(x)$ is a continuous non-decreasing bounded left function over $[0,1]$.

2. $\bar{\mu}(x)$ is a continuous non-increasing bounded right function over $[0,1]$.

3. $\mu(x) \leq \bar{\mu}(x), r \in[0,1]$. The crisp number $r$ is defined simply by $\underline{\mu}(r)=\bar{\mu}(r)=r, r \in[0,1]$.

Definition 2.3 [19]. If $\tilde{E}$ be the set of all fuzzy numbers, then $f(x)$ is a fuzzy function if $f: \mathbb{R} \rightarrow \tilde{E}$.

Definition 2.4 [20]. The fuzzy function process is denoted as A mapping $f: T \rightarrow \widetilde{E}$ for some interval $T \subseteq \tilde{E}$ and $r$-level set is represented by:

$$
[\tilde{f}(x)]_{r}=[\underline{f}(x ; r), \bar{f}(x ; r)], x \in K, r \in[0,1],
$$

where $K$ is the time interval. As representation types of the fuzzy set, the $r$-level sets of a fuzzy number are far more efficient than the above. Based on the resolution identity theorem, fuzzy sets can be represented by the families of their r-level sets.

Definition 2.5 [21]. Each function $f: X \rightarrow Y$ induces another function $\tilde{f}: F(X) \rightarrow F(Y)$. For each fuzzy interval $U$ in, it is defined by:

$$
\tilde{f}(U)(y)=\left\{\begin{array}{ll}
\operatorname{Sup}_{x \in f^{-1}(y)} U(x), & \text { if } y \in \operatorname{range}(f) \\
0 & \text { if } y \notin \operatorname{range}(f)
\end{array},\right.
$$

this is called the theory of the Zadeh extension..

\section{Description System of Fuzzy FDEs}

According to [22] and Section 2, consider the nonhomogeneous general system of FDEs with fuzzy initial conditions: 


$$
\left\{\begin{array}{c}
\tilde{y}^{\prime}{ }_{1}(t)=\tilde{f}_{1}\left(\tilde{y}_{1}(t), \ldots \tilde{y}_{n}(t)\right)+\tilde{g}_{1}(t), \\
\tilde{y}^{\prime}{ }_{2}(t)=\tilde{f}_{2}\left(\tilde{y}_{1}(t), \ldots \tilde{y}_{n}(t)\right)+\tilde{g}_{2}(t), \\
\cdot \\
\cdot \\
\tilde{y}_{n}^{\prime}(t)=\tilde{f}_{n}\left(\tilde{y}_{1}(t), \ldots \tilde{y}_{n}(t)\right)+\tilde{g}_{n}(t), \\
\tilde{y}_{1}(0)=\tilde{a}_{1}, \tilde{y}_{2}(0)=\tilde{a}_{2}, \ldots, \tilde{y}_{n}(0)=\tilde{a}_{n,}
\end{array}\right.
$$

where $\tilde{f}_{j}=\left[\underline{f}_{j}, \bar{f}_{j}\right]_{r}$ is the fuzzy function of the fuzzy variables $\tilde{y}_{j}=\left[\underline{y}_{i}, \bar{y}_{i}\right]_{r}$ and crisp variable $t$, the fuzzy Hukuhara-derivatives $\tilde{y}_{j}^{\prime}=\left[\underline{y}_{j}^{\prime}, \bar{y}_{j}^{\prime}\right]_{r}$ with fist order fuzzy differentiability [22].

The fuzzy initial conditions $\tilde{y}_{j}(0)=\left[\underline{y}_{j}(0), \bar{y}_{j}(0)\right]_{r}$ are triangular fuzzy numbers, and the nonhomogeneous terms $\tilde{g}_{j}=\left[\underline{g}_{j}, \bar{g}_{j}\right]_{r}$ for $j=1,2, \ldots n$. Following the fuzzy analysis in [22], we let $\tilde{Y}(t ; r)=\left[\tilde{y}_{1}(t ; r), \tilde{y}_{2}(t ; r), \ldots, \tilde{y}_{n}\left(t_{0} ; r\right)\right]$ be the solution of Eq. (1) in fuzzy form on $[0, T]$ such that by the theory of the Zadeh extension we have:

$$
\begin{gathered}
\underline{y}_{j}{ }^{\prime}(t ; r)=\min \left\{\tilde{f}_{j}\left(\tilde{y}_{1}(t ; r), \ldots \tilde{y}_{n}(t ; r)\right)+\underline{g}_{j}(t ; r) ; \tilde{y}_{j}(t ; r) \in\right. \\
\left.\left[\underline{y}_{j}(t ; r), \bar{y}_{j}(t ; r)\right]=\underline{f}_{j}(t, \tilde{Y}(t ; r))+\underline{g}_{j}(t, r)\right\} \\
\bar{y}_{j}{ }^{\prime}(t ; r)=\max \left\{\tilde{f}_{j}\left(\tilde{y}_{1}(t ; r), \ldots \tilde{y}_{n}(t ; r)\right)+, \bar{g}_{j}(t ; r) ; \tilde{y}_{j}(t ; r) \in\right. \\
\left.\left[\underline{y}_{j}(t ; r), \bar{y}_{j}(t ; r)\right]=\underline{f}_{j}(t, \tilde{Y}(t ; r))+\bar{g}_{j}(t, r)\right\}
\end{gathered}
$$

and

$$
\left\{\begin{array}{l}
f_{j}(t, \tilde{Y}(t ; r))=L_{j}(t, \underline{Y}(t ; r), \bar{Y}(t ; r))=L_{j}(t, \tilde{Y}(t ; r)) \\
\bar{f}_{j}(t, \tilde{Y}(t ; r))=U_{j}(t, \underline{Y}(t ; r), \bar{Y}(t ; r))=U_{j}(t, \tilde{Y}(t ; r))
\end{array}\right.
$$

According to the indicators mentioned above, the lower bound of system (1) is represented as follows;

$$
\left\{\begin{array}{l}
\underline{y}_{1}{ }^{\prime}(t ; r)=L_{1}(t, \tilde{Y}(t ; r))+\underline{g}_{1}(t ; r), \\
\underline{y}_{2}{ }^{\prime}(t ; r)=L_{2}(t, \tilde{Y}(t ; r))+\underline{g}_{2}(t ; r), \\
\cdot \\
\cdot \\
\underline{y}_{n}{ }^{\prime}(t ; r)=L_{n}(t, \tilde{Y}(t ; r))+\underline{g}_{n}(t ; r), \\
\underline{y}_{1}(0 ; r)=\underline{a}_{1}(r), \underline{y}_{2}(0 ; r)=\underline{a}_{2}(r), \ldots, \underline{y}_{n}(0 ; r)=\underline{a}_{n}(r),
\end{array}\right.
$$

and for the upper bound

$$
\left\{\begin{array}{c}
\bar{y}_{1}{ }^{\prime}(t ; r)=U_{1}(t, \tilde{Y}(t ; r))+\bar{g}_{1}(t ; r), \\
\bar{y}_{2}{ }^{\prime}(t ; r)=U_{2}(t, \tilde{Y}(t ; r))+\bar{g}_{2}(t ; r), \\
\cdot \\
\cdot \\
\bar{y}_{n}^{\prime}(t ; r)=U_{n}(t, \tilde{Y}(t ; r))+\bar{g}_{n}(t ; r), \\
\bar{y}_{1}(0 ; r)=\bar{a}_{1}(r), \ldots, \bar{y}_{n}(0 ; r)=\bar{a}_{n}(r) .
\end{array}\right.
$$

\section{Description of Fuzzy VIM}

The details of VIM to solve crisp differential equations have been introduced in [10-12]. The purpose of this section is to extend the fuzzy analysis of VIM to solve the system (1), from $[14,15]$ :

$\left\{\begin{array}{c}\underline{y}_{i+1,1}(t ; r)=\underline{y}_{i, 1}(t ; r)+\int_{0}^{t} \lambda(s ; r)\left\{\underline{y}_{i, 1}{ }^{\prime}(s ; r)-L_{1}\left(s, \widetilde{\widetilde{Y}_{i, 1}(s ; r)}\right)-\underline{g}_{1}(s ; r)\right\} d s \\ \underline{y}_{i+1,2}(t ; r)=\underline{y}_{i, 2}(t ; r)+\int_{0}^{t} \lambda(s ; r)\left\{\underline{y}_{i, 2}{ }^{\prime}(s ; r)-L_{2}\left(s, \widetilde{\widetilde{Y}}_{i_{2},}(s ; r)\right)-\underline{g}_{2}(s ; r)\right\} d s \\ \vdots \\ \underline{y}_{i+1, n}(t ; r)=\underline{y}_{i, n}(t ; r)+\int_{0}^{t} \lambda(s ; r)\left\{\underline{y}_{i, n}{ }^{\prime}(s ; r)-L_{n}\left(s, \widetilde{\widetilde{Y}_{i, n}(s ; r)}\right)-\underline{g}_{n}(s ; r)\right\} d s\end{array}\right.$

and for the upper bound

$\left\{\begin{array}{c}\bar{y}_{i+1,1}(t ; r)=\bar{y}_{i, 1}(t ; r)+\int_{0}^{t} \lambda(s ; r)\left\{\bar{y}_{i, 1}{ }^{\prime}(s ; r)-U_{1}\left(s, \widetilde{\widetilde{Y}_{i, 1}(s ; r)}\right)-\bar{g}_{1}(s ; r)\right\} d s \\ \bar{y}_{i+1,2}(t ; r)=\bar{y}_{i, 2}(t ; r)+\int_{0}^{t} \lambda(s ; r)\left\{\bar{y}_{i, 2}{ }^{\prime}(s ; r)-U_{2}\left(s, \widetilde{Y}_{i, 2}(s ; r)\right)-\bar{g}_{2}(s ; r)\right\} d s \\ \vdots \\ \bar{y}_{i+1, n}(t ; r)=\bar{y}_{i, n}(t ; r)+\int_{0}^{t} \lambda(s ; r)\left\{\bar{y}_{i, n}{ }^{\prime}(s ; r)-U_{n}\left(s, \widetilde{\widetilde{Y}_{i, n}(s ; r)}\right)-\bar{g}_{n}(s ; r)\right\} d s\end{array}\right.$

for $i=0,1,2, \ldots, m$, and $j=1,2, \ldots, n$. The general Lagrange multiplier $\lambda(t, s)$ which can be optimally identified via variational theory [10]. $\widetilde{Y}_{i, j}$ is deemed a restricted variation. i.e $\delta \tilde{Y}_{i, j}=0$. According to [15], the value of $\lambda(s ; \eta)=-1$ related to Eqs. (7-8), then Eqs. (7-8) becomes as follows:

$$
\left\{\begin{array}{c}
\underline{y}_{i+1,1}(t ; r)=\underline{y}_{i, 1}(t ; r)-\int_{0}^{t}\left\{\underline{y}_{i, 1}{ }^{\prime}(s ; r)-\underline{f}_{1}\left(s, \underline{Y}_{i, 1}(s ; r)\right)-\underline{g}_{1}(s ; r)\right\} d s \\
\underline{y}_{i+1,2}(t ; r)=\underline{y}_{i, 2}(t ; r)-\int_{0}^{t}\left\{\underline{y}_{i, 2}{ }^{\prime}(s ; r)-\underline{f}_{2}\left(s, \underline{Y}_{i, 2}(s ; r)\right)-\underline{g}_{2}(s ; r)\right\} d s \\
\cdot \\
\cdot \\
\underline{y}_{i+1, n}(t ; r)=\underline{y}_{i, n}(t ; r)-\int_{0}^{t}\left\{\underline{y}_{\underline{i n},}{ }^{\prime}(s ; r)-\underline{f}_{n}\left(s, \underline{Y}_{i, n}(s ; r)\right)-\underline{g}_{n}(s ; r)\right\} d s
\end{array}\right.
$$

and for the upper bound

$$
\left\{\begin{array}{c}
\bar{y}_{i+1,1}(t ; r)=\bar{y}_{i, 1}(t ; r)-\int_{0}^{t}\left\{\bar{y}_{i, 1}{ }^{\prime}(s ; r)-\bar{f}_{1}\left(s, \bar{y}_{i, 1}(s ; r)\right)-\bar{g}_{1}(s ; r)\right\} d s \\
\bar{y}_{i+1,2}(t ; r)=\bar{y}_{i, 2}(t ; r)-\int_{0}^{t}\left\{\bar{y}_{i, 2}{ }^{\prime}(s ; r)-\bar{f}_{2}\left(s, \bar{y}_{i, 2}(s ; r)\right)-\bar{g}_{2}(s ; r)\right\} d s \\
\vdots \\
\cdot \\
\bar{y}_{i+1, n}(t ; r)=\bar{y}_{i, n}(t ; r)-\int_{0}^{t}\left\{\bar{y}_{i, n}{ }^{\prime}(s ; r)-\bar{f}_{n}\left(s, \bar{y}_{i, n}(s ; r)\right)-\bar{g}_{n}(s ; r)\right\} d s
\end{array}\right.
$$

where the valued of the initial guessing $\tilde{y}_{0, j}(t ; r)$ that satisfy the initial conditions in Eq. (1). Consequently from [15], the exact solutions $\tilde{F}_{j}(t ; r)$ of system (1) may be obtained by

$$
\tilde{F}_{j}(t ; r)=\lim _{i \rightarrow 1} \tilde{y}_{i}(t ; r)
$$

\section{Fuzzy Linear HIV Model Solution and Analysis}

The diseases that affect human immune system can be identified as the HIV infection, where the protective cell $\mathrm{CD} 4+\mathrm{T}$ cell are being actively depleted. This will lead to 
immunosuppression and defense against opportunistic infections. When the infection occurs, HIV is passed and the cell molecule CD4 $+\mathrm{T}$ is bound to the HIV receptor in the protection cycle. HIV with bind to that cell and insert genetic material into $\mathrm{CD} 4+\mathrm{T}$ cells and then transforms ribonucleic acid (RNA) into deoxyribonucleic acid (DNA) using the enzyme known as reverse transcriptase to transform the CD4+T cell structure into HIV.CD4 + T-cell serves as the transmitter of a signal by cytokines to the other immune cell. In general, cell CTLs respond to this message and are designed to remove cells from HIV. The powerful immune system in this case reduces the rate of depletion of the CD4 + T-cell and vice versa. Diminishing $\mathrm{CD} 4+\mathrm{T}$ cell molecules is not able to react or act against pathogen from bursting during proliferation, and the immune system becomes weaker [23]. The HIV patients are now moving to patients with AIDS. Zarei et al [23] proposed fuzzy linear structure underneath.

$$
\left\{\begin{array}{l}
\tilde{x}^{\prime}(t)=\tilde{l}-\tilde{q} \tilde{x}(t)-\tilde{c} \tilde{v}(t), \\
\tilde{v}^{\prime}(t)=\tilde{p} \tilde{v}(t)-\tilde{a} \tilde{z}(t), \\
\tilde{z}^{\prime}(t)=\tilde{h} \tilde{x}(t)-\tilde{T} \tilde{v}(t), \\
\tilde{x}(0)=\tilde{x} 0, \tilde{v}(0)=\tilde{v}_{0}, \tilde{z}(0)=\tilde{z}_{0},
\end{array}\right.
$$

where the variables and perimeters in model (12) are defined in [24] also the dynamic behavior and details of system (12) is given in [23]. Under section four, the VIM formula of system (12) is given by

$$
\begin{array}{r}
\tilde{x}_{i+1}(t ; r)=\tilde{x}_{i}(t ; r)-\int_{0}^{t}\left\{\tilde{x}_{i}{ }^{\prime}(s ; r)-[]_{r}+[\tilde{q}]_{r} \tilde{x}_{i}(s ; r)+[\tilde{c}]_{r} \tilde{x}_{i j}(s ; r)\right\} d s, \\
\tilde{v}_{i+1}(t ; r)=\tilde{v}_{i}(t ; r)-\int_{0}^{t}\left\{\tilde{v}_{i}{ }^{\prime}(s ; r)-[\tilde{p}]_{r} \tilde{v}_{i}(s ; r)+\right. \\
\left.[\tilde{a}]_{r} \tilde{z}_{i}(s ; r)\right\} d s, \tilde{z}_{i+1}(t ; r)=\tilde{z}_{i}(t ; r)-\int_{0}^{t}\left\{\tilde{z}_{i}{ }^{\prime}(s ; r)-\right. \\
\left.[\tilde{h}]_{r} \tilde{x}_{i}(s ; r)+[\tilde{T}]_{r} \tilde{v}_{i}(s ; r)\right\} d s, \\
\tilde{x}(0 ; r)=\left[\tilde{x}_{0}\right]_{r}, \tilde{v}(0 ; r)=\left[\tilde{v}_{0}\right]_{r}, \tilde{z}(0 ; r)=\left[\tilde{z}_{0}\right]_{r} .
\end{array}
$$

From VIM in Eq. (13) we obtain the following results:

\subsection{Immune Cell-Level Dynamics and Low Patient Viral Load}

Figure 1: (a), (b) and (c) display immune system levels that are CD4+T cells, CTLs, and HIV viral stress for patients with a low immune system at a time interval of between 0 and 2000 days. (The dark color shows the highest chance to improve the immunity, while the light dark color shows the low chance to improve the immunity). Figure 1 (a) shows the slowly decline of $\mathrm{CD} 4+\mathrm{T}$ cell level at the beginning. The curves show that the number of $\mathrm{CD} 4+\mathrm{T}$ cells is rapidly decreasing. The curves show that the number of $\mathrm{CD} 4+\mathrm{T}$ cells is rapidly decreasing. The curves show that there are rapid decreases in the number of CD4+ $\mathrm{T}$ cells after slowly incline and the progression to AIDS have the highest possibility to occur. Figure 1 (b) shows that there is very slow increment in the HIV viral load at the beginning. Besides, if we observe the curves there are highest probability that HIV viral load increases rapidly after slowly increase. Moreover, we can say that the speed of HIV virus increases corresponding to the speed depletion of CD4+ T cells. This is due to proliferation of new HIV cell by bursting the CD4+ T cell. Figure 1 (c) shows the level of CTLs gradually increases when the CD4+ T cell gradually decrease at early stage but decline at the latter stage which is due to virus induce the impairment to CD4+ T cell.

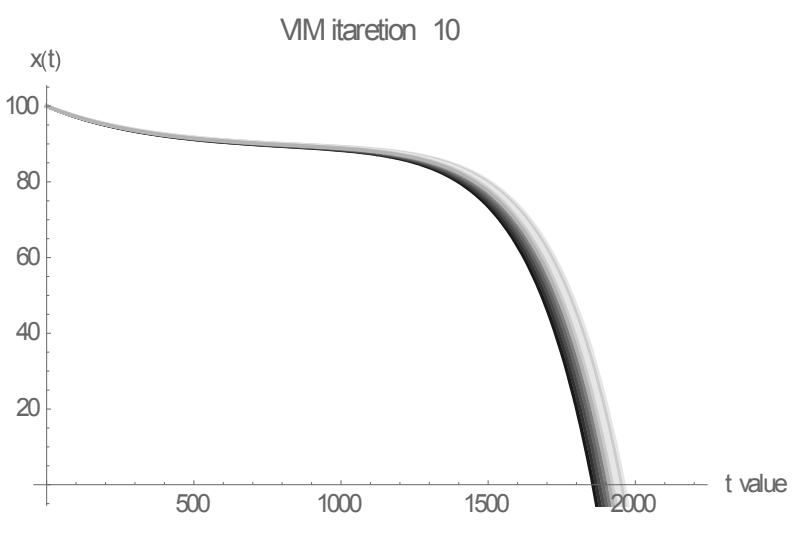

(a)

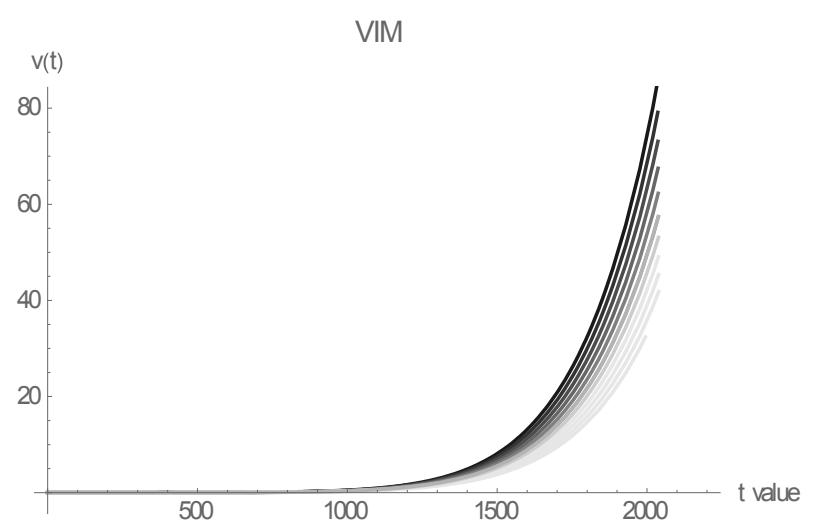

(b)

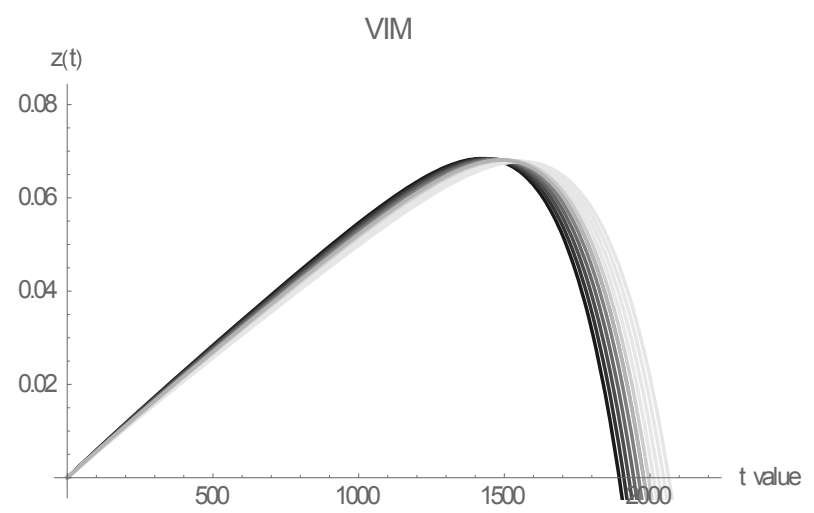

(c)

Figure 1. (a) CD4+ T cell level, (b) HIV viral load, (c) CTLs level in low immune system patient for $t \in[0,2000]$ days 


\subsection{Immune Cell-Level Dynamics and Moderate Patient Viral Load}

The degree of CD4 $+\mathrm{T}$ cell decreases slowly but at a reasonable rate as shown in Figure 2(a). In addition, the number of $\mathrm{CD} 4+\mathrm{T}$ cells is likely to decrease in the moderate rate later after a progressive reduction. Figure 2(b) shows that at a later stage of HIV infection, the level of viral load still gradually increases at a reasonable rate. In this case the lasting immune response also depends on the CD4 $+\mathrm{T}$ and HIV viral load levels in Figure 2(c). Figure 2(c) indicates a modest response rate. The chance is also mild in this case.

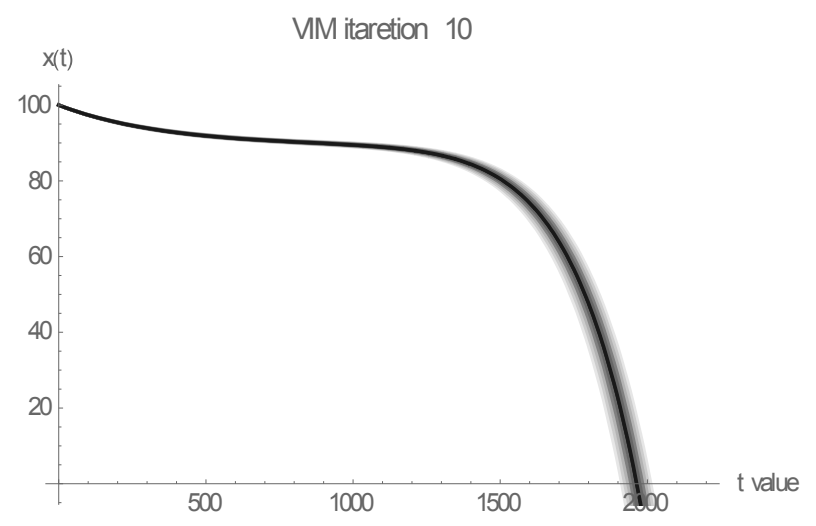

(a)

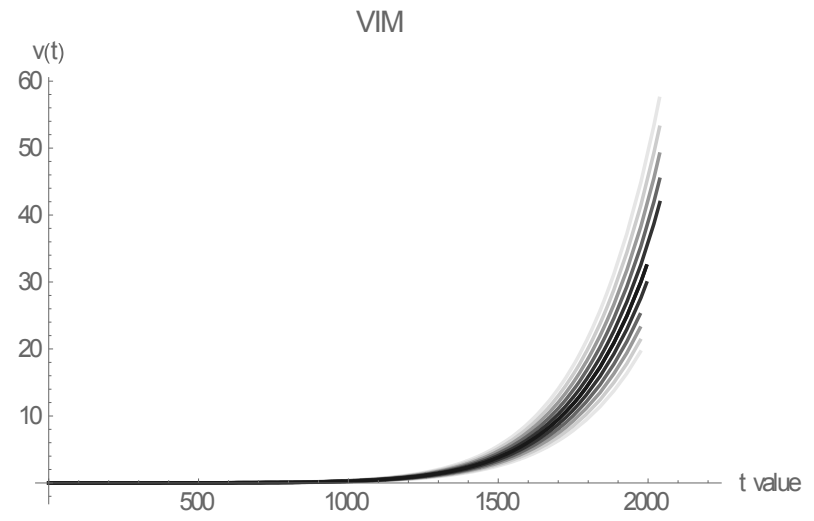

(b)

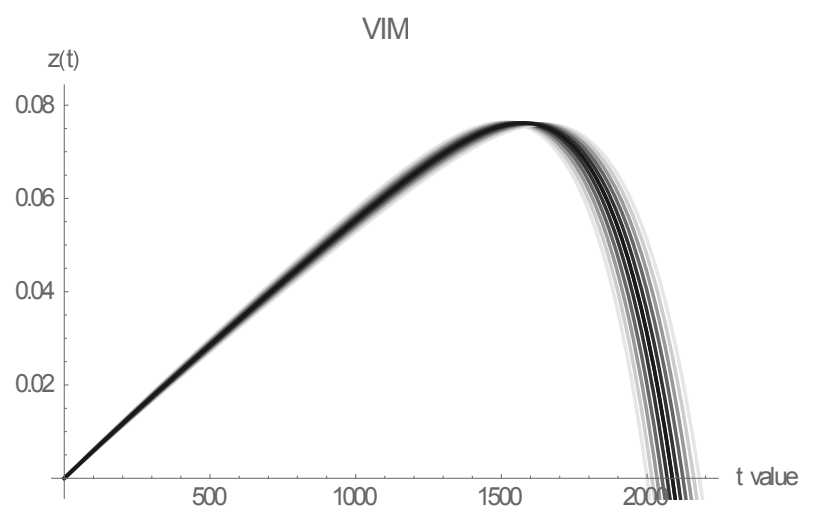

(c)

Figure 2. (a) CD4+ T cell level, (b) HIV viral load, (c) CTLs level in moderate immune system patient $t \in[0,2000]$ days

\subsection{Immune Cell-Level Dynamics and High Patient Viral Load}

The steady decrease of CD4 + T cell over a 2000 time period can be seen in Figure 3 (a). The curves indicate that the CD4 $+\mathrm{T}$ cell is most likely to continue to decline slowly. Furthermore, the gradual decrease of the CD4+T cell contributes to a slow development of AIDS. Figure 3(b) shows that HIV viral load rates are gradually increasing. HIV viral load can gradually increase. There are strong prospects. The high probability of a longer CTL cell is shown in Figure 3 (c). The minimum virus deficiency is demonstrated by the $\mathrm{CD} 4+\mathrm{T}$ cell.

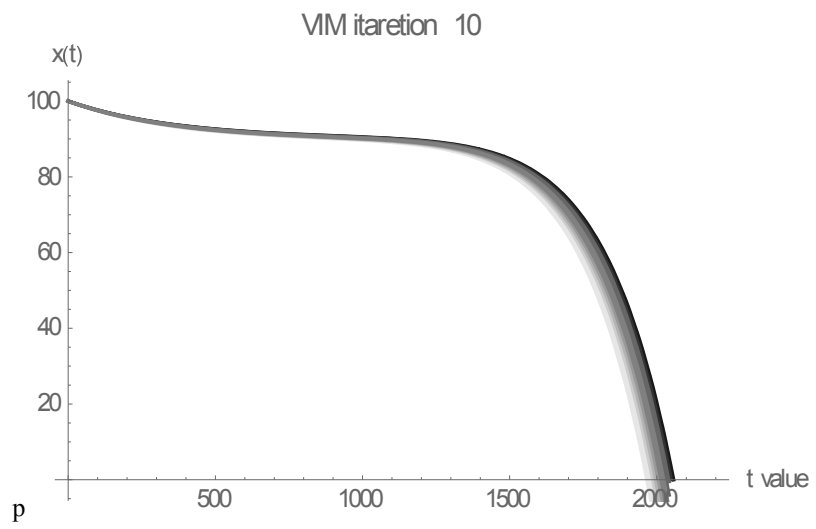

(a)

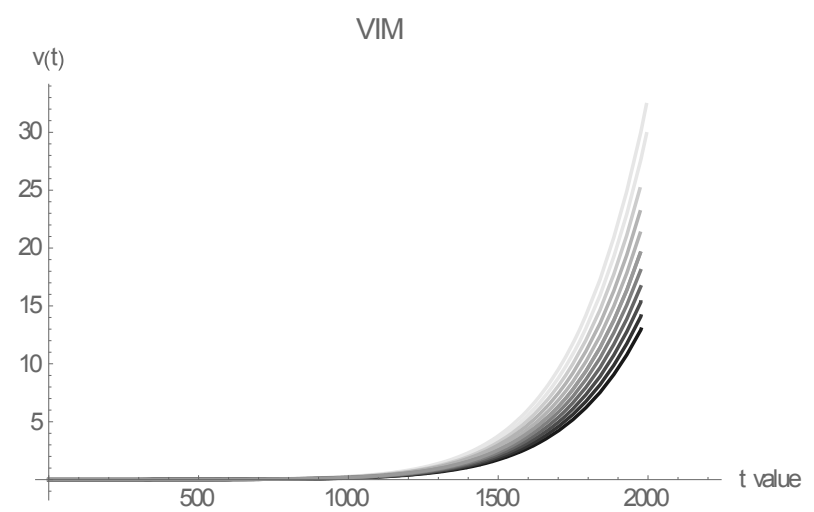

(b)

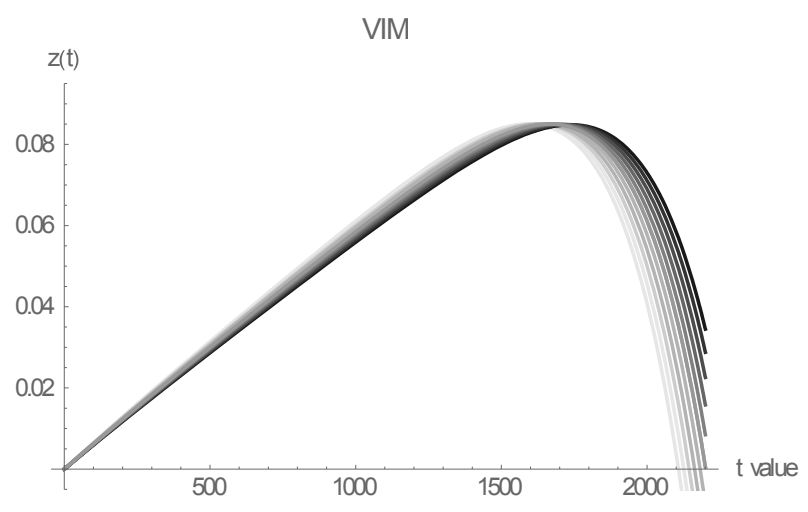

(c)

Figure 3. (a) CD4+ T cell level, (b) HIV viral load, (c) CTLs level in high immune system patient for $t \in[0,2000]$ Days 


\subsection{Weighted Center of Gravity (WCOG)}

This technique provides a crisp value dependent on the fuzzy defined center of gravity. The total area used to describe the combined control action in the fuzzy membership functions distribution is divided into a variety of sub-areas. The area and the center of gravity or center of each sub-area are measured and all these sub-areas are then defuzzified for a discreet fuzzy set [23]. We illustrate the WCOG in Figures $4 \mathrm{a}, \mathrm{b}$ and $\mathrm{c}$ as bellow:

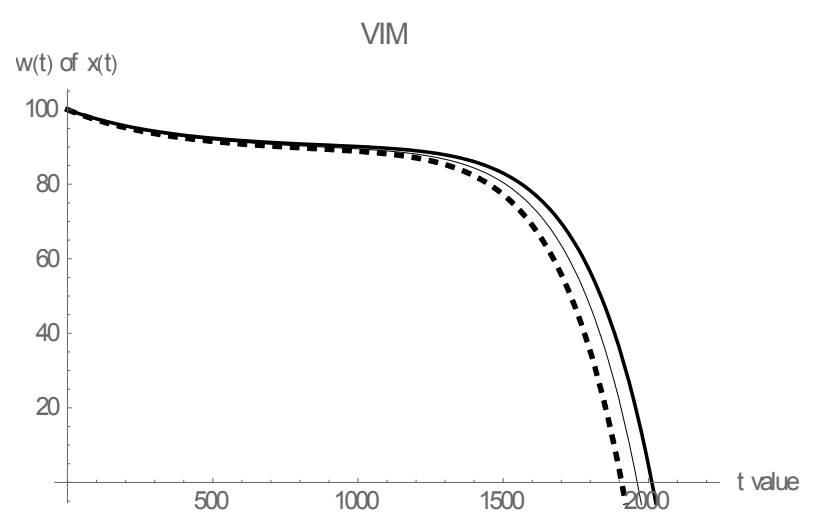

(a)

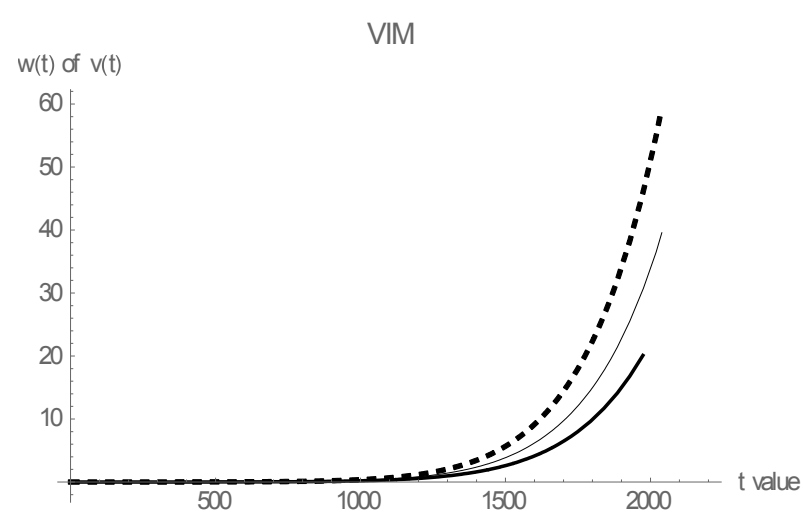

(b)

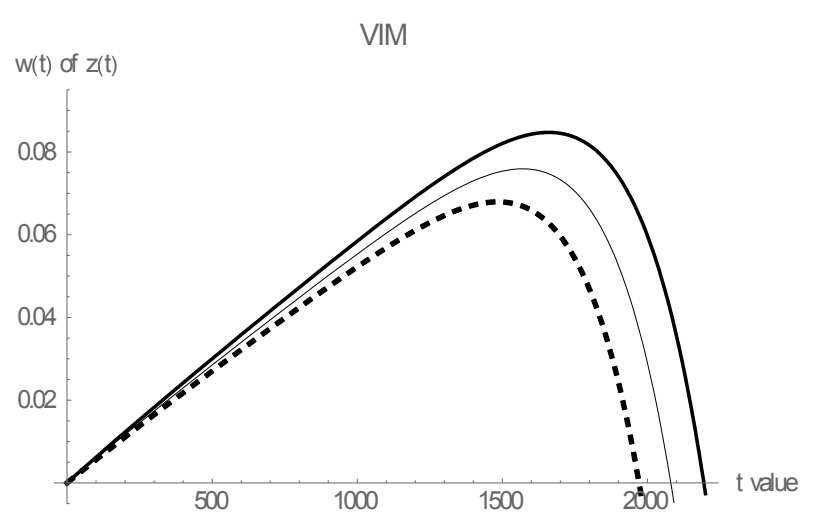

(c)

Figure 4. The WCOG of CD4+ T-cells level (a), the viral load (b) and CTLs level (c) versus time in patients $\mathrm{L}(\ldots), \mathrm{M}(-)$, and $\mathrm{H}(-)$ for $t \in[0,2000]$ days

\subsection{The r-Cut Indicating the Level of Immune Cell and Viral Load}

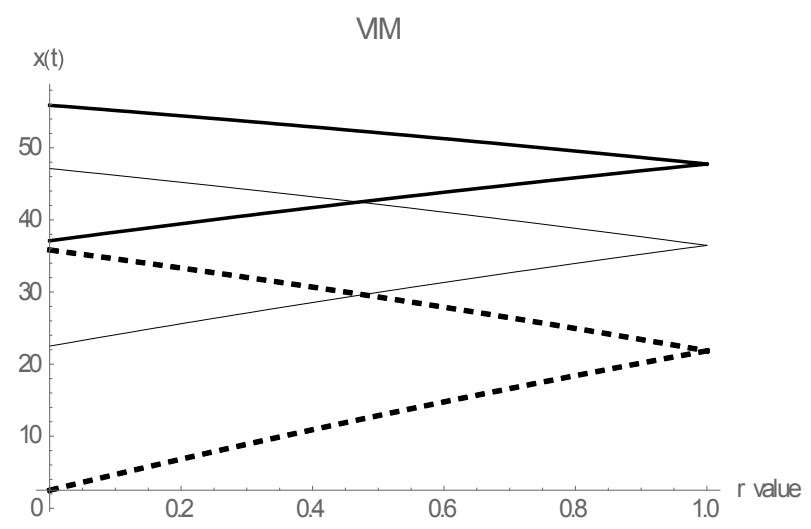

(a)

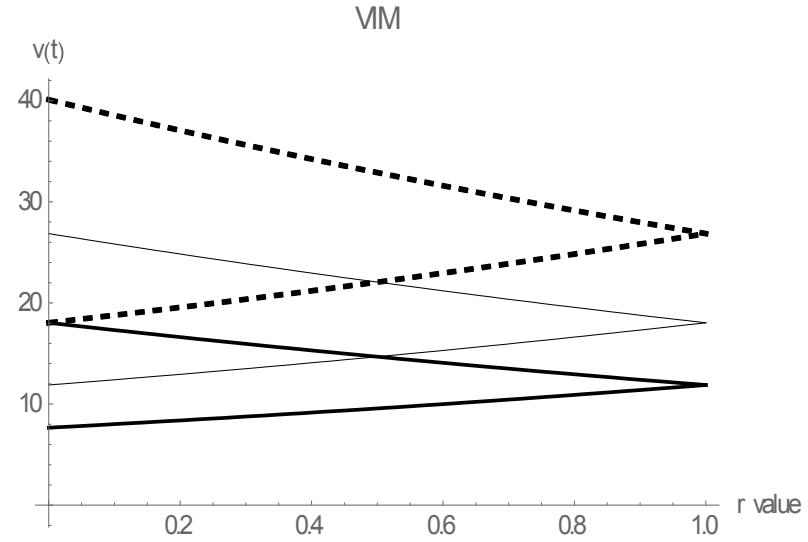

(b)

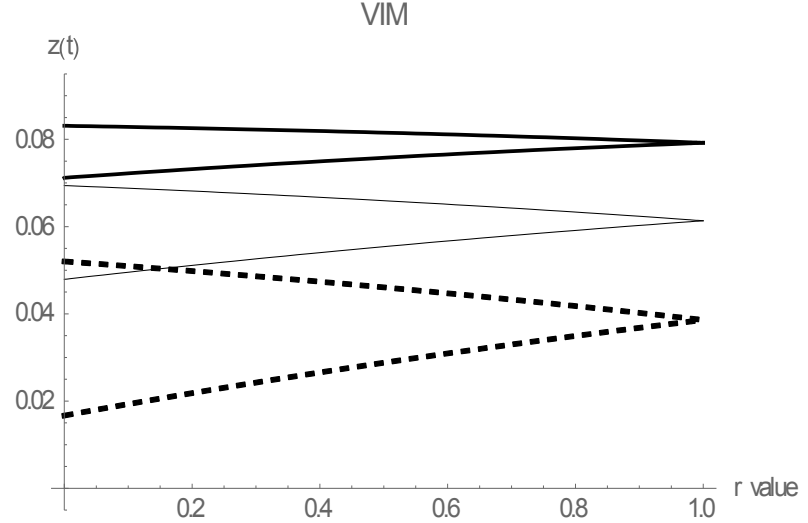

(c)

Figure 5. (a) r-cut level for CD4+ $\mathrm{T}$ cell (b) r-cut level for HIV viral load (c) r-cut level for CTLs cell in the form of triangular fuzzy number solution

The r-cut value against number of cells is drawn at $t=1850$ by the plot of Figure 5(a), (b), (c). Nevertheless, the amount of these cell is different for the high immune patient when $r=1$ is more when $r=0$. The number of CD4 $+\mathrm{T}$ cells between low and high in the case of a moderate immune system. This shows that the 
number of cells CD4+T in $t=1850$ in strong immune patients is higher compared with patients with poor immune strength. The increase in HIV viral load is decreased by the number of CD4+T cells, as stated in 3.2. The number of HIV viruses dependent on the r-cut is shown in Figure 5(b).

\section{Conclusions}

We proposed the approximate method known as VIM to describe the mathematical model of fuzzy HIV dynamic. VIM is reformulated and analyzed in term of fuzzy domain in the linear fuzzy HIV infection model. The recreates appear that the fuzzy HIV model can be utilized in portraying the vulnerability resistant cell level and the viral stack because of existing patients with the distinctive quality of immunization level. The obtained results by VIM shows that the method series solution obey the properties in the form of the triangular fuzzy number.

\section{Acknowledgements}

The authors acknowledge financial support from the School of Mathematical Sciences, Universiti Sains Malaysia, Penang and the enormous support provided throughout the research.

\section{REFERENCES}

[1] Tomasiello, S,. Khattri S. K., and Awrejcewicz, J. (2017) 'Differential Quadrature-Based Simulation Of A Class Of Fuzzy Damped Fractional Dynamical Systems', International Journal of Numerical Analysis And Modeling, Vol. 14, No. 1, pp. 63-75.

[2] Perelson, A.S. 1989. Modeling the interaction of HIV with immune system, Mathematical and Statistical Approaches to AIDS Epidemiology (Castillo-Chavez ed.), Lect. Notes in Biomath. 83, Springer, Berlin, 350-370.

[3] Perelson, A.S., Kirschner, D.E., De Boer, R. 1993. Dynamics of HIV infection of CD4+T cells, Mathematical Biosciences, 114(1), 81-125.

[4] Ali, N., Zaman, G. 2016. Asymptotic behavior of HIV-1 epidemic model with infinite distributed intracellular delays. Springer Plus, 5(1), 324.

[5] Omer, A., and Omer, O. (2013) 'A Pray and Pretdour Model with Fuzzy Initial Values', Hacettepe Journal of Mathematics and Statistics, Vol. 41, No. 3, pp. 387-395.

[6] Tapaswini, S., and Chakravery, S. (2013) 'Numerical solution of fuzzy arbitrary order predator-prey equations', Applications and Applied Mathematics, Vol. 8, No. 1, pp.647-673.
[7] El Naschie, M.S. (2005) 'From experimental quantum optics to quantum gravity via a fuzzy Kahler manifold', Chaos Solution and Fractals, Vol. 25, No. 5, pp.969-977.

[8] Ali, V. K., and Ali, A. H. (2012) 'Fuzzy Modeling and Control of HIV Infection', Computational and Mathematical Methods in Medicine, Vol. 2012, pp. 1-17.

[9] Jameel, A.F., Anakira, N.R., Alomari, A.K., Alsharo, D. M., and Saaban, A. (2019) 'New semi-analytical method for solving two point nth order fuzzy boundary value problem, Int. J. Mathematical Modelling and Numerical Optimisation, Vol. 9, No. 1, pp. 21-31.

[10] He, J. H. (1998) 'Approximate solution of nonlinear differential equations with convolution product nonlinearities', Computer Methods in Applied Mechanics and Engineering, Vol. 167, No 1-2, pp. 69-73.

[11] Bildik, N., and Ali, K. (2006) 'The use of variational iteration method, differential transform method and Adomian decomposition method for solving different types of nonlinear partial differential equations', International Journal of Nonlinear Sciences and Numerical Simulation, Vol. 7, No. 1, pp. 65-75.

[12] Xu, L.(2006) 'Variational Principles For Coupled Nonlinear Schrodinger Equations, Physics Letters A, Vol. 359, No. 6, pp. 627-629.

[13] Anakira, N. R., Shather, A. H., Jameel, A. F., Alomari, A. K., Saaban, A. (2018) 'Direct solution of uncertain bratu initial value problem', International Journal of Electrical and Computer Engineering, vol. 9. No.6, pp. 221-240.

[14] Allahviranloo,T., Abbasbandy, S., and Behzadi, S., (2014) 'Solving nonlinear fuzzy differential equations by using fuzzy variational iteration method', Soft Computing, Vol. 18 , No. 11, pp. 2191-2200.

[15] Abbasbandy, S., Allahviranloo,T., Darabi, P., and Sedaghatfar, O. (2011) ' Variational Iteration Method For Solving $\mathrm{n}$-th Order Fuzzy Differential Equations', Mathematical and Computational Applications, Vol. 16, No. 4, pp. 819-829.

[16] Rishi, V. L., Rui, Z., Asha, D. V., and Bing, X. (2012) 'CD4 ${ }^{+} \mathrm{T}$ Cells: Differentiation and Functions, Clinical and Developmental Immunology, Vol.2012, pp. 1-12.

[17] Dubois, D. and Prade, H. (1982) 'Towards fuzzy differential calculus, Part 3: Differentiation', Fuzzy Sets and Systems, Vol. 8, No. 3, pp.225-233.

[18] Seikkala, S. (1987) 'On the fuzzy initial value problem', Fuzzy Sets Systems, Vol. 24, No. 3, pp. 319-330.

[19] Fard, O.S. (2009) 'An iterative scheme for the solution of generalized system of linear fuzzy differential equations', World Applied Sciences Journal, Vol. 7, No. 12, pp.159711604.

[20] Fard, O.S. (2009) 'An iterative scheme for the solution of generalized system of linear fuzzy differential equations', World Applied Sciences Journal, Vol. 7, No. 12, pp.159711604. 
[21] Zadeh, L.A. (2005) 'Toward a generalized theory of uncertainty', Information Sciences, Vol. 172, No. 2, pp.140.

[22] A. F. Jameel, N. R. Anakira, A. K. Alomari, I. Hashim, M. A. Shakhatreh, Numerical solution of nth order fuzzy initial value problems by six stages, Journal of nonlinear science and applications. 9(2) 627-640, 2016.

[23] Muhammad, B. R., and Normah, M. (2017) 'Numerical solution for linear fuzzy differential equation in HIV infection', eProceedings Chemistry. Vol. 2, pp. 318-324.

[24] Zarei, H., Kamyad, A.V., and Heydari, A.A. (2012). 'Fuzzy modelling and control of HIV infection', Computational and Mathematical Methods in Medicine, Vol. 2012, pp. 1-17.

[25] Shanmugasundaram, N., Ganesh, E.N., Kumar, N. "Estimation of power analysis in WLAN infrastructure" International Journal of Engineering and Technology(UAE), 2018, 7(2), pp. 198-200

[26] Shanmugasundaram, N., Sushita, K., Kumar, S.P., Ganesh, E.N. "Genetic algorithm-based road network design for optimising the vehicle travel distance" International Journal of Vehicle Information and Communication Systems, 2019, 4(4), pp. 355-374 